\title{
Virulence Characterization of Single-Zoospore Isolates of Sclerospora graminicola from Pearl Millet
}

\author{
R. P. Thakur, B. Pushpavathi, and V. P. Rao, Genetic Resources and Enhancement Program, International Crops \\ Research Institute for the Semi-Arid Tropics (ICRISAT), Patancheru 502 324, Andhra Pradesh, India
}

\begin{abstract}
Thakur, R. P., Pushpavathi, B., and Rao, V. P. 1998. Virulence characterization of single-zoospore isolates of Sclerospora graminicola from pearl millet. Plant Dis. 82:747-751.

Sclerospora graminicola, the causal agent of downy mildew in pearl millet, is well-known for variation in its virulence pattern. Nine single-zoospore isolates ( $\mathrm{Sg}$ 026-Z-1 to Sg 026-Z-9) derived from an oosporic isolate Sg 026 from a pearl millet $F_{1}$ hybrid cultivar Nath 4209 grown in a farmer's field in a village, Veelad, in Maharashtra state, India, and three controls (Sg 026, Field-1, and Field-2) were evaluated for their virulence in two experimental runs in a greenhouse. The isolates were maintained on pot-grown seedlings of a highly susceptible pearl millet line, 7042S, in a greenhouse through asexual (sporangial) generations. Pot-grown seedlings of six pearl millet potential differential lines/cultivars (7042S, NHB 3, MBH 110, ICMH 451, $843 \mathrm{~B}$, and $852 \mathrm{~B})$ were spray-inoculated with a sporangial suspension $\left(5 \times 10^{5}\right.$ sporangia ml-1 $)$ and maintained in a greenhouse at $25 \pm 2^{\circ} \mathrm{C}$. Data were recorded for latent period (days) and disease incidence $(\%)$, from which a virulence index (incidence $\times$ latent period ${ }^{-1}$ ) was calculated to quantify disease-causing potential of isolates. Results indicated significant variation in latent period, incidence, and virulence index among isolates. The isolates were classified into four distinct pathotype groups based on their virulence indices on six pearl millet lines. Because of the significant variation for virulence in the $S$. graminicola population infecting Nath 4209 , it is recommended that the hybrid be regularly monitored for downy mildew infection in farmers' fields, and be replaced by a resistant cultivar that is genetically unrelated to the parental lines of Nath 4209. This will help delay or avoid development of downy mildew epidemics and the resulting heavy loss to pearl millet farmers in the region.
\end{abstract}

Variation in virulence patterns of Sclerospora graminicola (Sacc.) Schroet., the causal agent of downy mildew of pearl millet (Pennisetum glaucum (L.) R. Br.) is well-established $(1,2,5,10,13,17-19)$. The fungus is largely heterothallic $(5,7)$, but homothallism may also exist (7). These characteristics of the fungus make it highly variable like its host, pearl millet, which is a highly outcrossing crop species. Our understanding of the mechanisms underlying the evolution of pathogenic variation in S. graminicola is limited by the lack of genetically well-defined host differentials and the lack of molecular genetic techniques to understand the pearl millet- $S$. graminicola interactions. During the past 2 years, we have generated information on molecular markers for downy mildew resistance genes (6) and developed a DNA fingerprinting technique to detect genetic variations in the pathogen populations (9).

The first evidence of pathogenic variation in downy mildew of pearl millet was

Corresponding author: R. P. Thakur

E-mail address: r.thakur@cgnet.com

ICRISAT Journal Article No. 2057

Accepted for publication 8 March 1998.

Publication no. D-1998-0427-02R

(C) 1998 The American Phytopathological Society reported in 1973 when an $F_{1}$ hybrid cultivar (HB 3) was found to be resistant at Mysore, but susceptible at some other locations in India (3). Nene and Singh (8) interpreted this variation as being due to the existence of races in the pathogen. Shetty and Ahmad (10) reported that two distinct races from Mysore and Gulbarga were easily differentiated on HB 3. Results from the International Pearl Millet Downy Mildew Nursery (IPMDMN), as determined by disease reaction on a set of cultivars after several years of operation at diverse locations in India and West Africa, clearly indicated the existence of distinct virulences in the pathogen populations $(13,15)$. The pathogen populations from West Africa were generally more virulent than those from India $(1,6,13,15)$. Evolution of a host-specific pathotype was clearly demonstrated by Singh and Singh (14) at Durgapura, Rajasthan, where NHB 3 , a highly susceptible hybrid, showed resistance after not being grown for 4 years, but subsequently became susceptible again when grown continuously.

Surveys in farmers' fields and results of field trials showed increased incidence of downy mildew on MBH 110, a pearl millet hybrid cultivar, in Maharashtra over a period of a few years, indicating the selection of host cultivar-specific virulence in the $S$. graminicola population $(16,17)$. More examples of host-specificity toward com- mercial $\mathrm{F}_{1}$ hybrid cultivars MLBH 104 and Nath 4209 (Nath Seed Company Ltd., Aurangabad, India) were found in our recent surveys (R. P. Thakur and others, unpublished data).

Evolution of host-specific virulence from a field population of $S$. graminicola was demonstrated by serial passage of asexual spores for 5 to 10 generations on two pearl millet lines (19). Molecular genetic analysis has further confirmed the evolution of host-specific pathotypes of $S$. graminicola in six pearl millet lines, using mini- and microsatellites (9). These results again indicate the highly variable nature of the pathogen and its ability to match new resistance genes in the host cultivar. In the present study, our objective was to examine the extent of virulence diversity among single-zoospore isolates derived from the original oospore inoculum obtained from a commercial pearl millet $\mathrm{F}_{1}$ hybrid cultivar, Nath 4209, which recently became susceptible to downy mildew.

\section{MATERIALS AND METHODS}

Plant material. A susceptible pearl millet genotype, $7042 \mathrm{~S}$, was used for obtaining single-zoospore isolates from the original oosporic sample (Sg 026) collected from the commercial pearl millet hybrid cultivar, Nath 4209, from a farmer's field in Veelad village in Maharashtra state, India. Surface-sterilized seeds were planted in 5-cm-diameter pots filled with sterilized soil, 1 seed per pot. Seedlings were inoculated at the coleoptile stage using the syringe inoculation method (12).

Single-zoospore isolates. Fresh sporangia were harvested into cold water $\left(4^{\circ} \mathrm{C}\right)$, from the ninth asexual (sporangial) generation of isolate $\mathrm{Sg} 026$. A diluted (2 to 3 sporangia/field of microscope) suspension of these sporangia was prepared. This suspension was kept at $25^{\circ} \mathrm{C}$ for about $30 \mathrm{~min}$ to allow the release of zoospores from sporangia. A small amount $(0.5 \mathrm{ml})$ of zoospore suspension was spread uniformly over the surface of sterile water agar medium $(1 \%)$ in petri plates and the excess suspension was drained off. Single, wellisolated zoospores were marked on water agar using a dummy objective under the microscope $(10 x)$. Single zoospores were picked up with the help of a flat-tipped needle and transferred onto the emerging coleoptile of pearl millet seedlings (7042S) grown in small pots. Pots were then covered with polyethylene bags and incubated 
overnight at $20^{\circ} \mathrm{C}$. The pots were then transferred to a greenhouse at $25 \pm 2{ }^{\circ} \mathrm{C}$. Plants were observed daily for symptoms. Of 250 inoculated seedlings, only 12 showed symptoms. From these, only 9 seedlings survived; these were maintained separately in polyacrylic isolation chambers in a greenhouse. The isolates were designated $\mathrm{Sg}$ 026-Z-1 through Sg 026-Z-9.

Test for virulence. Pot-grown seedlings of six host differentials (7042S, NHB 3, MBH 110, ICMH 451, 843B, and 852B) were spray-inoculated with a sporangial suspension $\left(5 \times 10^{5}\right.$ sporangia $\left.\mathrm{ml}^{-1}\right)$ of each zoospore isolate. The parental isolate $\mathrm{Sg} 026$ and two International Crops Research Institute for the Semi-Arid Tropics (ICRISAT) Patancheru field isolates (Field-1 and Field-2) from a mixture of $7042 \mathrm{~S}$ and NHB 3 were used as controls. All 12 isolates were used in experiment 1, while only 7 of these were used in experiment 2 (adequate inoculum of 5 isolates was not available). The experiment was conducted in a randomized block design with three replications, and at least 100 seedlings were evaluated per replication. Data were recorded for latent period (time in days from inoculation to sporulation), and the number of infected seedlings. For latent-period data, recording began 3 days after inoculation and continued until day 11. "Latent period" was expressed as the number of days when about $50 \%$ of the infected seedlings in a replication showed sporulation. Disease incidence (\% of infected seedlings) was recorded on day 14 after inoculation.

For the purpose of this study, we define "virulence" as the relative capacity of an isolate to infect a host line; it is measured qualitatively as virulent or avirulent. We define "aggressiveness" as the capacity of a virulent isolate to produce a particular amount of disease in a host line; it is measured quantitatively (\% disease inci- dence). Virulence index, the quantitative measure of virulence, was calculated as disease incidence $(\%) \times$ latent $_{\text {period }}{ }^{-1}$ (17). We consider virulence index different from pathogenic fitness index, which was described by Thakur and Shetty (18) as a function of latent period, percentage incidence, sporangial production per unit leaf area, and oospore production potential of the isolate. The isolates were grouped into different pathotypes based on their virulence indices on the potential host differentials.

Statistical analysis. Means and least significant differences were estimated, and analysis of variance was carried out, with the GENSTAT statistical package (Rothamsted Experiment Station, Herpenden, UK) to determine significant effects of isolates, host lines, and their interactions on latent period, downy mildew incidence, and virulence index. Average Linkage Cluster analysis was done using the Euclidean test to determine the similarity among the isolates and to classify them into pathotype groups based on virulence index.

\section{RESULTS}

Variation in virulence. The zoospore isolates infected five of the six pearl millet lines in both experiments and therefore were considered virulent (Table 1). Isolates Sg 026-Z-2, -Z-3, -Z-5, and -Z-7 failed to cause disease on MBH 110, nor did isolate Sg 026-Z-5 cause disease on $843 \mathrm{~B}$, and so these were considered avirulent on these host lines. The other isolates were virulent

Table 2. Analysis of variance for percent downy mildew incidence (DM), latent period in days (LP), and virulence index $(\mathrm{VI})$ for experiment 1

\begin{tabular}{|c|c|c|c|c|c|c|}
\hline \multirow[b]{2}{*}{ Source of variation } & \multicolumn{2}{|r|}{ DM } & \multicolumn{2}{|c|}{ LP } & \multicolumn{2}{|r|}{ VI } \\
\hline & df & $\mathbf{M S}^{\mathbf{a}}$ & df & MS & df & MS \\
\hline Replication & 2 & 63.89 & 2 & 0.09 & 2 & 2.46 \\
\hline Isolate (I) & 11 & $2,874.42 * * * \mathrm{~b}$ & 11 & $9.90 * * *$ & 11 & $122.11 * * *$ \\
\hline Host differential $(\mathrm{H})$ & 5 & $51,904.81 * * *$ & 5 & $24.98 * * *$ & 5 & $1,590.57 * * *$ \\
\hline $\mathrm{I} \times \mathrm{H}$ & 55 & $973.98^{* * * *}$ & 48 & $2.76 * * *$ & 55 & $32.98 * *$ \\
\hline Error & 142 & 66.97 & 112 & 1.12 & 142 & 1.88 \\
\hline
\end{tabular}

${ }^{a}$ Mean square.

b **, *** $=$ significant at $P<0.01$ and $P<0.001$, respectively.

Table 3. Analysis of variance for percent downy mildew incidence (DM), latent period in days (LP), and virulence index (VI) for experiment 2

\begin{tabular}{|c|c|c|c|c|c|c|}
\hline \multirow[b]{2}{*}{ Source of variation } & \multicolumn{2}{|r|}{ DM } & \multicolumn{2}{|c|}{ LP } & \multicolumn{2}{|c|}{ VI } \\
\hline & df & $\mathbf{M S}^{\mathbf{a}}$ & df & MS & df & MS \\
\hline Replication & 2 & 88.39 & 2 & 0.07 & 2 & 1.59 \\
\hline Isolate (I) & 6 & $3,397.51 * * * \mathrm{~b}$ & 6 & $13.18^{* * *}$ & 6 & $158.77 * * *$ \\
\hline Host differential $(\mathrm{H})$ & 5 & $27,508.79 * * *$ & 5 & $13.72 * * *$ & 5 & $768.84 * * *$ \\
\hline $\mathrm{I} \times \mathrm{H}$ & 30 & $1,093.80 * * *$ & 26 & $3.88 * * *$ & 30 & $36.80 * *$ \\
\hline Error & 82 & 15.24 & 65 & 0.12 & 82 & 0.38 \\
\hline
\end{tabular}

a Mean square.

b **, *** $=$ significant at $P<0.01$ and $P<0.001$, respectively.

Table 1. Downy mildew incidence $(\%)^{\mathrm{a}}$ on a set of pearl millet host differentials induced by single-zoospore isolates of Sclerospora graminicola under greenhouse conditions

\begin{tabular}{|c|c|c|c|c|c|c|c|c|c|c|c|c|c|c|}
\hline \multirow[b]{3}{*}{ S. graminicola isolate } & \multicolumn{14}{|c|}{ Host differential in experiments 1 and $2^{b}$} \\
\hline & \multicolumn{2}{|c|}{$7042 S$} & \multicolumn{2}{|c|}{ NHB 3} & \multicolumn{2}{|c|}{ MBH 110} & \multicolumn{2}{|c|}{ ICMH 451} & \multicolumn{2}{|c|}{ 843B } & \multicolumn{2}{|c|}{ 852B } & \multicolumn{2}{|c|}{ Mean } \\
\hline & 1 & $2^{c}$ & 1 & 2 & 1 & 2 & 1 & 2 & 1 & 2 & 1 & 2 & 1 & 2 \\
\hline $\mathrm{Sg} 026$ & 92 & 92 & 95 & 92 & 1 & 1 & 8 & 9 & 13 & 12 & 62 & 65 & 45 & 45 \\
\hline Sg 026-Z-1 & 98 & 93 & 94 & 98 & 2 & 2 & 5 & 3 & 49 & 50 & 89 & 87 & 56 & 55 \\
\hline Sg 026-Z-2 & 96 & $\ldots$ & 92 & $\ldots$ & 0 & $\ldots$ & 10 & $\ldots$ & 4 & $\ldots$ & 94 & $\ldots$ & 49 & $\ldots$ \\
\hline $\mathrm{Sg} \mathrm{026-Z-3}$ & 96 & 93 & 92 & 95 & 0 & 0 & 15 & 13 & 4 & 3 & 92 & 85 & 50 & 48 \\
\hline Sg 026-Z-4 & 96 & $\ldots$ & 89 & $\ldots$ & 1 & $\ldots$ & 19 & $\ldots$ & 55 & $\ldots$ & 84 & $\ldots$ & 58 & $\ldots$ \\
\hline Sg 026-Z-5 & 52 & 53 & 48 & 55 & 0 & 0 & 4 & 2 & 0 & 0 & 57 & 54 & 27 & 27 \\
\hline Sg 026-Z-6 & 43 & 45 & 39 & 39 & 3 & 2 & 4 & 5 & 26 & 30 & 62 & 66 & 30 & 31 \\
\hline $\mathrm{Sg} 026-\mathrm{Z}-7$ & 89 & $\ldots$ & 82 & $\ldots$ & 0 & $\ldots$ & 13 & $\ldots$ & 35 & $\ldots$ & 76 & $\ldots$ & 49 & $\ldots$ \\
\hline Sg 026-Z-8 & 99 & 96 & 99 & 100 & 1 & 1 & 21 & 23 & 87 & 88 & 86 & 86 & 65 & 66 \\
\hline Sg 026-Z-9 & 99 & $\ldots$ & 100 & $\ldots$ & 7 & $\ldots$ & 16 & $\ldots$ & 63 & $\ldots$ & 93 & $\ldots$ & 63 & $\ldots$ \\
\hline Field-1 & 92 & $\ldots$ & 100 & $\ldots$ & 0 & $\ldots$ & 15 & $\ldots$ & 3 & $\cdots$ & 11 & $\ldots$ & 37 & $\cdots$ \\
\hline Field-2 & 95 & 91 & 98 & 97 & 0 & 0 & 10 & 9 & 5 & 6 & 6 & 7 & 36 & 35 \\
\hline Mean & 87 & 80 & 86 & 82 & 1 & 1 & 12 & 9 & 29 & 27 & 68 & 64 & & \\
\hline
\end{tabular}

a Mean of three replications, based on total and diseased plants.

${ }^{\mathrm{b}}$ Least significant difference $(P<0.001)$. Experiment 1: isolate $\times$ differential $=21.9$, isolate $=8.9$, differential $=6.3$; experiment 2 : isolate $\times$ differential $=$ 10.89 , isolate $=4.44$, differential $=4.12$.

${ }^{\mathrm{c}}$ Only 7 of the 12 isolates were evaluated in experiment 2. 
on all host lines, but the two control field isolates were also avirulent on MBH 110.

Variation in aggressiveness. Numbers of plants in each host line infected by different isolates varied considerably. This ranged from $1 \%$ incidence for isolates $\mathrm{Sg}$ 026-Z-4 and -Z-8 on MBH 110 , to $100 \%$ for Sg 026-Z-9 on NHB 3 (Table 1). The field isolates and $\mathrm{Sg} 026$ were also highly aggressive on NHB 3, inducing 92 to $100 \%$ disease in both experiments. All 12 isolates were generally highly aggressive on $7042 \mathrm{~S}$ and NHB 3; Sg 026 and their zoospore derivatives were also relatively highly aggressive on $852 \mathrm{~B}$, while low to moderate aggressiveness was observed for all isolates on MBH 110 and ICMH 451, and low to very high on $843 \mathrm{~B}$. The analysis of variance indicated highly significant $(P<$ 0.001 ) effects of isolates, host lines, and their interaction on incidence in both experiment 1 (Table 2) and experiment 2 (Table 3).
Variation in latent period. The mean latent period varied from 5 days for isolate Sg 026-Z-9 and Field-1 on NHB 3 to 11 days for Sg 026-Z-8 on MBH 110, and 8.8 days for Sg 026-Z-6 on 843B (Table 4). Even on the known susceptible host lines, such as 7042S and NHB 3, the latent periods were highly variable with different isolates as well as for the field isolate. Across isolates, the latent period was greatest on $\mathrm{MBH} 110$ (8.1 and 8.3 days), and least on NHB 3 (5.9 and 6.3 days), while across the host lines it was greatest for isolate $\mathrm{Sg} \mathrm{026-Z-5} \mathrm{(8.5} \mathrm{and} 8.4$ days) and least for isolate Field-1 (5.9 days). The mean squares for isolate, host lines, and their interaction were highly significant $(P$ $<0.001$ ) for latent period (Tables 2 and 3).

Variation in virulence index. Virulence index was quite variable, and ranged from 0 to 20 in the two experiments (Table 5). Virulence index was generally greater for most isolates on 7042S and NHB 3, mod- erate on 852B, and lower on MBH 110, ICMH 451, and 843B. Across host lines, isolate $\mathrm{Sg}$ 026-Z-8 had the greatest virulence index (12.34 and 12.20) and $\mathrm{Sg} 026-$ Z-5 the lowest (3.28 and 3.30), while across isolates it was highest on NHB 3 (15.36 and 14.22) and lowest on MBH 110 (0.16 and 0.11$)$. Even with the highly susceptible host lines (7042S and NHB 3), the virulence index varied from 5.2 to 20.0. Virulence index was highly influenced by isolates, host lines, and their interaction (Table 2).

Pathotype grouping. The 12 isolates in experiment 1 were classified into four major pathotype groups based on virulence index (Fig. 1A). The two field isolates and $\mathrm{Sg} 026$ formed the first group; five singlezoospore isolates, Sg 026-Z-1 to -Z-4 and $-Z-7$, formed the second group; Sg 026-Z-5 and $-\mathrm{Z}-6$ formed the third group; and $\mathrm{Sg}$ 026-Z-8 and -Z-9 formed the fourth group. In experiment 2,7 of these isolates fol-

Table 4. Mean latent period (days) ${ }^{\mathrm{a}}$ on six pearl millet lines induced by zoospore isolates of Sclerospora graminicola under greenhouse conditions

\begin{tabular}{|c|c|c|c|c|c|c|c|c|c|c|c|c|c|c|}
\hline \multirow[b]{3}{*}{ S. graminicola isolate } & \multicolumn{14}{|c|}{ Host differential in experiments 1 and $2^{b}$} \\
\hline & \multicolumn{2}{|c|}{ 7042S } & \multicolumn{2}{|c|}{ NHB 3} & \multicolumn{2}{|c|}{ MBH 110} & \multicolumn{2}{|c|}{ ICMH 451} & \multicolumn{2}{|c|}{ 843B } & \multicolumn{2}{|c|}{ 852B } & \multicolumn{2}{|c|}{ Mean } \\
\hline & 1 & $2^{c}$ & 1 & 2 & 1 & 2 & 1 & 2 & 1 & 2 & 1 & 2 & 1 & 2 \\
\hline Sg 026 & 6.3 & 6.3 & 5.5 & 5.5 & 6.0 & 6.0 & 7.7 & 7.5 & 7.0 & 7.2 & 7.3 & 7.3 & 6.6 & 6.6 \\
\hline Sg 026-Z-1 & 6.3 & 6.3 & 5.2 & 5.5 & 7.0 & 7.5 & 10.5 & 10.5 & 7.2 & 7.5 & 7.2 & 7.3 & 7.2 & 7.4 \\
\hline Sg 026-Z-2 & 5.7 & $\ldots{ }^{\mathrm{d}}$ & 5.8 & $\ldots$ & $\ldots$ & $\ldots$ & 6.7 & $\ldots$ & 8.0 & $\ldots$ & 6.8 & $\ldots$ & 6.6 & $\ldots$ \\
\hline Sg 026-Z-3 & 6.2 & 6.0 & 6.0 & 6.0 & $\ldots$ & $\ldots$ & 7.7 & 7.7 & 6.0 & 6.3 & 6.3 & 6.5 & 6.4 & 6.5 \\
\hline Sg 026-Z-4 & 6.2 & $\ldots$ & 6.2 & $\ldots$ & 8.0 & $\ldots$ & 7.2 & $\ldots$ & 7.3 & $\ldots$ & 6.8 & $\ldots$ & 7.0 & $\ldots$ \\
\hline Sg 026-Z-5 & 8.0 & 8.0 & 8.8 & 8.8 & & . & 9.0 & 9.0 & & & 8.3 & 8.0 & 8.5 & 8.4 \\
\hline Sg 026-Z-6 & 7.8 & 7.7 & 7.3 & 7.5 & 8.5 & 8.5 & 6.7 & 6.7 & 8.8 & 8.5 & 7.2 & 7.5 & 7.7 & 7.7 \\
\hline Sg 026-Z-7 & 5.8 & $\ldots$ & 5.8 & $\ldots$ & $\ldots$ & $\ldots$ & 7.7 & $\ldots$ & 6.0 & & 6.8 & 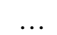 & 6.4 & $\ldots$ \\
\hline Sg 026-Z-8 & 5.2 & 5.3 & 5.0 & 5.0 & 11.0 & 11.0 & 6.3 & 6.3 & 5.5 & 5.5 & 5.5 & 5.5 & 6.4 & 6.4 \\
\hline Sg 026-Z-9 & 5.0 & $\ldots$ & 5.0 & $\ldots$ & 8.0 & $\ldots$ & 7.3 & $\ldots$ & 7.2 & $\ldots$ & 5.7 & $\ldots$ & 6.4 & $\ldots$ \\
\hline Field-1 & 5.3 & $\ldots$ & 5.0 & $\ldots$ & $\ldots$ & $\ldots$ & 7.2 & $\ldots$ & 5.0 & $\ldots$ & 7.0 & $\ldots$ & 5.9 & $\ldots$ \\
\hline Field-2 & 5.7 & 5.5 & 5.3 & 5.5 & $\ldots$ & $\ldots$ & 7.3 & 7.5 & 5.8 & 5.8 & 6.7 & 6.5 & 6.2 & 6.2 \\
\hline Mean & 6.1 & 6.4 & 5.9 & 6.3 & 8.1 & 8.3 & 7.6 & 7.9 & 6.9 & 6.8 & 6.8 & 6.9 & & \\
\hline
\end{tabular}

a Mean of three replications.

${ }^{\mathrm{b}}$ Least significant difference $(P<0.001)$. Experiment 1 : isolate $\times$ differential $=2.93$, isolate $=1.20$, differential $=0.84$; experiment 2 : isolate $\times$ differential $=0.96$, isolate $=0.39$, differential $=0.36$.

${ }^{\mathrm{c}}$ Only 7 of the 12 isolates were evaluated in experiment 2.

d No symptoms observed.

Table 5. Virulence index ${ }^{a}$ (downy mildew incidence $(\%) \times$ latent period $^{-1}$ ) caused by zoospore isolates of Sclerospora graminicola on six pearl millet lines under greenhouse conditions

\begin{tabular}{|c|c|c|c|c|c|c|c|c|c|c|c|c|c|c|}
\hline \multirow[b]{3}{*}{ S. graminicola isolate } & \multicolumn{14}{|c|}{ Host differential in experiments 1 and $2^{b}$} \\
\hline & \multicolumn{2}{|c|}{$7042 S$} & \multicolumn{2}{|c|}{ NHB 3} & \multicolumn{2}{|c|}{ MBH 110} & \multicolumn{2}{|c|}{ ICMH 451} & \multicolumn{2}{|c|}{ 843B } & \multicolumn{2}{|c|}{ 852B } & \multicolumn{2}{|c|}{ Mean } \\
\hline & 1 & $2^{\mathrm{c}}$ & 1 & 2 & 1 & 2 & 1 & 2 & 1 & 2 & 1 & 2 & 1 & 2 \\
\hline $\mathrm{Sg} 026$ & 14.6 & 14.5 & 17.3 & 16.8 & 0.1 & 0.2 & 1.1 & 1.2 & 1.7 & 1.7 & 8.4 & 8.8 & 6.43 & 7.21 \\
\hline Sg 026-Z-1 & 15.6 & 14.7 & 18.3 & 17.8 & 0.3 & 0.3 & 0.6 & 0.3 & 6.8 & 6.6 & 12.5 & 11.9 & 9.03 & 8.59 \\
\hline Sg 026-Z-2 & 16.9 & & 15.8 & 170 & 0.0 & $\ldots$ & 1.6 & $\ldots$ & 0.5 & & 13.8 & $2 x$ & 8.11 & \\
\hline $\mathrm{Sg} \mathrm{026-Z-3}$ & 15.5 & 15.4 & 15.3 & 15.9 & 0.0 & 0.0 & 1.9 & 1.7 & 0.7 & 0.5 & 14.7 & 13.1 & 8.00 & 7.76 \\
\hline Sg 026-Z-4 & 15.5 & $\ldots$ & 14.5 & & 0.1 & $\ldots$ & 2.7 & & 7.5 & & 12.3 & & 8.80 & $\ldots$ \\
\hline Sg 026-Z-5 & 6.6 & 6.6 & 5.3 & 6.2 & 0.0 & 0.0 & 0.7 & 0.3 & 0.0 & 0.0 & 7.1 & 6.7 & 3.28 & 3.30 \\
\hline Sg 026-Z-6 & 5.5 & 5.8 & 5.3 & 5.2 & 0.3 & 0.2 & 0.6 & 0.8 & 3.0 & 3.6 & 8.7 & 8.8 & 3.90 & 4.08 \\
\hline Sg 026-Z-7 & 15.2 & & 14.1 & & 0.0 & $\ldots$ & 1.7 & & 6.2 & & 11.2 & & 8.10 & \\
\hline Sg 026-Z-8 & 19.1 & 17.8 & 19.8 & 20.0 & 0.1 & 0.1 & 3.5 & 3.6 & 15.9 & 16.0 & 15.7 & 15.6 & 12.34 & 12.20 \\
\hline Sg 026-Z-9 & 19.7 & $\ldots$ & 20.0 & $\ldots$ & 0.9 & $\ldots$ & 2.1 & $\ldots$ & 8.9 & $\ldots$ & 16.4 & $\ldots$ & 11.34 & $\ldots$ \\
\hline Field-1 & 17.3 & $\ldots$ & 20.0 & $\ldots$ & 0.0 & $\ldots$ & 2.0 & $\ldots$ & 0.7 & $\ldots$ & 1.5 & $\ldots$ & 6.92 & $\ldots$ \\
\hline Field-2 & 16.8 & 16.6 & 18.4 & 17.6 & 0.0 & 0.0 & 1.4 & 1.2 & 0.9 & 1.0 & 0.9 & 1.2 & 6.40 & 6.29 \\
\hline Mean & 14.88 & 13.09 & 15.36 & 14.22 & 0.16 & 0.11 & 1.67 & 1.29 & 4.40 & 4.21 & 10.26 & 9.44 & & \\
\hline
\end{tabular}

a Mean of three replications.

${ }^{\mathrm{b}}$ Least significant difference $(P<0.001)$. Experiment 1: isolate $\times$ differential $=3.69$, isolate $=1.52$, differential $=1.07$; experiment 2 : isolate $\times$ differential $=1.71$, isolate $=0.70$, differential $=0.65$.

${ }^{\mathrm{c}}$ Only 7 of the 12 isolates were evaluated in experiment 2. 
lowed the same pattern, except Sg 026 and Field-2, which were separated into two groups (Fig. 1B).

\section{DISCUSSION}

The results indicate significant variation for virulence, aggressiveness, latent period, and virulence index among single-zoospore isolates of $S$. graminicola. Our results support the earlier findings on pathogenic variation among oospore isolates (17) and sporangial isolates (18), and provides further information at the zoospore level. In the earlier study (19), the influence of hostdirected virulence selection in a pathogen population was demonstrated using a bulk field isolate of the pathogen and passing it through several asexual generations (sporangia-zoospores) on the same host lines. In this study, we have demonstrated how single-zoospore derivatives from a bulk isolate obtained from a single host line (commercial hybrid cultivar in this case) could be variable for virulence. The extent of variation is significant and has epidemiological significance for the pearl millet downy mildew pathosystem. The 12 isolates in experiment 1 and 7 in experiment 2 , based on virulence index, were classified into four pathogenicity groups, indicating significant variability in the parental isolate $\mathrm{Sg} 026$.

The parental isolate was collected from the commercial pearl millet cultivar, Nath 4209, a private sector hybrid, being widely grown in several districts of Maharashtra-one of the largest pearl millet-growing states in India, where hybrid cultivars are grown on more than $60 \%$ of its pearl millet area. During the 1993 rainy season survey, a downy mildew incidence of 27 to $53 \%$ was observed in farmers' fields (4). Virulence analysis of the $S$. graminicola population from Nath 4209 indicates adaptation to several other pearl millet culti- vars and lines, indicating the existence of several matching virulence genes to other downy mildew resistance genes. Several years ago, another popular private sector $F_{1}$ hybrid, MBH 110, succumbed to downy mildew, which resulted in withdrawal of this hybrid from cultivation. In 1993, yet another private sector hybrid, MLBH 104, became very susceptible to downy mildew (4). Preliminary virulence analysis of isolates from these hybrids indicated the occurrence of new pathotypes specific to these cultivars (16). The present virulence analysis of single-zoospore isolates from Nath 4209 has provided further evidence of intrapopulation pathogenic variability. In addition to host-specificity in pathogen, certain single-zoospore isolates of the population ( $\mathrm{Sg} \mathrm{026)}$ have been highly aggressive on other pearl millet cultivars, such as 7042S, NHB 3, 843B, and 852B. The fact that none of these isolates showed high virulence on $\mathrm{MBH} 110$ indicates that the virulence in this population is quite different from the population that infects $\mathrm{MBH}$ 110. Also, 852B is resistant to the ICRISAT Patancheru field populations of the pathogen, but highly susceptible to these single-zoospore isolates. ICRISATbred hybrid ICMH 451 exhibited moderate resistance to all the isolates, including the field isolates, but indications from field surveys are that the hybrid has begun showing susceptibility in Gujarat state, where it is being grown in a large area ( $R$. P. Thakur, unpublished data).

Evolution of host-specific pathotypes in S. graminicola has been well demonstrated by virulence analysis using potential host differentials $(1,2)$, an international downy mildew nursery $(11,13)$, an international downy mildew virulence nursery (15), and virulence selection studies $(17,19)$. These results have been further confirmed by molecular studies using quantitative trait
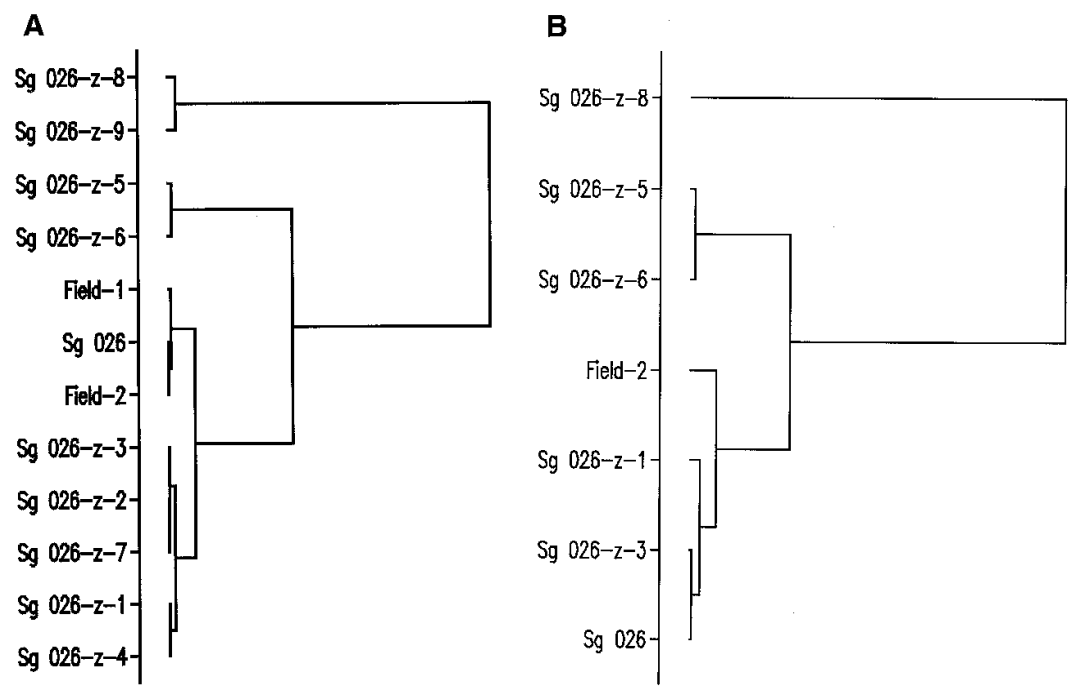

Fig. 1. Classification of zoospore isolates of Sclerospora graminicola based on average linkage cluster analysis of virulence index using Euclidean test. (A) 12 isolates in experiment 1 and (B) 7 isolates in experiment 2.

loci analysis (6), and restriction fragment length polymorphism and random amplified polymorphic DNA analyses (9). Because of the highly variable nature of the pathogen and rapid adaptation to new host cultivars, particularly to genetically uniform single-cross $F_{1}$ hybrids, several such hybrids (NHB 3, BJ 104, MBH 110, MLBH 104, and Nath 4209) have succumbed to the disease during the past few years in India, and the process continues. In contrast to $F_{1}$ hybrids, the genetically heterogeneous, open-pollinated cultivars WC-C75 and ICTP 8203 have shown durable resistance during their commercial cultivation on a large scale for a number of years (4). $F_{1}$ hybrid cultivars, despite being short-lived for downy mildew resistance, are preferred by farmers because of their uniformity, early maturity, and relatively higher yield than open-pollinated cultivars. A well-planned strategy to monitor virulence changes in the pathogen and resistance in host cultivars, and identification and incorporation of novel resistance genes using marker-assisted selection breeding and their proper deployment, will help reduce the chances of epidemics and losses from downy mildew in pearl millet.

\section{LITERATURE CITED}

1. Ball, S. L. 1983. Pathogenic variability of downy mildew (Sclerospora graminicola) on pearl millet-host cultivar reactions to infection by different pathogen isolates. Ann. Appl. Biol. 102:257-264.

2. Ball, S. L., Pike, D. J., and Burridge, C. Y. 1986. Characterization of populations of Sclerospora graminicola. Ann. Appl. Biol. 108:519-526.

3. Bhat, S. S. 1973. Investigations on the biology and control of Sclerospora graminicola on Bajra. Ph.D. Thesis. Department of Post Graduate Studies and Research in Botany, University of Mysore, Mysore, India.

4. ICRISAT. 1996. Page 76 in: ICRISAT Asia Regional Report 1995. ICRISAT Patancheru 502325 , A. P., India.

5. Idris, M. O., and Ball, S. L. 1984. Inter- and intracontinental sexual compatibility in Sclerospora graminicola. Plant Pathol. 33:219223.

6. Jones, E. S., Liu, C. J., Gale, M. D., Hash, C. T., and Witcombe, J. R. 1995. Mapping quantitative trait loci for downy mildew resistance in pearl millet. Theor. Appl. Genet. 91:448456.

7. Michelmore, R. W., Pawar, M. N., and Williams, R. J. 1982. Heterothallism in Sclerospora graminicola. Phytopathology 72:1368-1372.

8. Nene, Y. L., and Singh S. D. 1976. Downy mildew and ergot of pearl millet. PANS 22:366-385.

9. Sastry, J. G., Ramakrishna, W., Sivaramkrishnan, S., Thakur, R. P., Gupta, V. S., and Ranjekar, P. K. 1995. DNA fingerprinting detects genetic variability in the pearl millet downy pathogen (Sclerospora graminicola). Theor. Appl. Genet. 91:856-861.

10. Shetty, H. S., and Ahmad, R. 1981. Physiologic specialization in Sclerospora graminicola. Indian Phytopathol. 34:307-309.

11. Singh, S. D. 1995. Downy mildew of pearl millet. Plant Dis. 79:545-550.

12. Singh, S. D., and Gopinath, R. 1985. A seedling inoculation technique for detecting downy mildew resistance in pearl millet. Plant 
Dis. 69:582-584.

13. Singh, S. D., King, S. B., and Werder, J. 1993. Downy mildew disease of pearl millet. ICRISAT Inf. Bull. 37. ICRISAT Patancheru, A. P. 502 324, India.

14. Singh, S. D., and Singh, G. 1987. Resistance to downy mildew in pearl millet hybrid NHB 3. Indian Phytopathol. 40:178-180.

15. Thakur, R. P. 1995. Status of international sorghum anthracnose and pearl millet downy mildew virulence nurseries. Pages 75-92 in:
Disease Analysis through Genetics and Biotechnology: Interdisciplinary Bridges to Improve Sorghum and Millet Crops. J. F. Leslie and R. A. Frederiksen, eds. Iowa State University Press, Ames.

16. Thakur, R. P., and Rao, V. P. 1996. Evidence for a new virulent pathotype in Sclerospora graminicola on pearl millet. (Abstr.) Indian J. Mycol. Plant Pathol. 26:133.

17. Thakur, R. P., and Rao, V. P. 1997. Variation in virulence and aggressiveness among pathotypes of Sclerospora graminicola on pearl millet. Indian Phytopathol. 50:41-47.

18. Thakur, R. P., and Shetty, K. G. 1993. Variation in pathogenicity among single-oospore isolates of Sclerospora graminicola, the causal organism of downy mildew in pearl millet. Plant Pathol. 42:715-721.

19. Thakur, R. P., Shetty, K. G., and King, S. B. 1992. Selection for host-specific virulence in asexual populations of Sclerospora graminicola. Plant Pathol. 41:626-632. 